\title{
A Study on Super Vertex Graceful Graphs
}

\author{
N.Murugesan \\ Post graduate and Research Dept. of \\ Mathematics, \\ Government Arts College, (Autonomous), \\ Coimbatore- 18, \\ Tamil Nadu
}

\author{
R.Uma \\ Dept. of Mathematics, \\ SreeSaraswathi Thyagaraja College, \\ (Autonomous), Pollachi
}

\begin{abstract}
In this paper a brief discussion is made on the super vertex graceful graphs. In particular the order and size plays vital role in labelling the graphs. Also an analysis is made on the order of the complete bipartite graphs under super vertex graceful map.
\end{abstract}

\section{AMS Classification $05 \mathrm{C} 78$}

\section{Keywords}

Complete graphs, Cycles, Complete bipartite graphs, Graceful graphs, Super vertex graceful graphs.

\section{INTRODUCTION}

A graph labelling serve as useful models for a broad range of applications such as coding theory, X-ray, crystallography, etc. Graceful

labelling was introduced by Rosa in 1967.Since then many types of labelling namely Harmonious labelling, total magic labelling, edge graceful labelling, odd edge graceful labelling, etc were emerged as given in Dynamic survey [7]. Sin Min Lee has introduced super vertex graceful labelling [15]. N.Murugesan, R.Uma [10] have analysed the super vertex gracefulness of complete bipartite graphs. Also they have discussed the amalgamation of graphs under graceful mapping and Fibonacci graceful labelling[9]. Also, N.Murugesan, R.Uma[13] have analysed the role of order and size of cycles, crowns, wheels under super vertex graceful mapping. In this paper an analysis is made on the order size and degree of the graphs under super vertex graceful map.

\section{DEFINITION}

\subsection{Graceful labelling}

Let $\mathrm{G}=(\mathrm{V}, \mathrm{E})$ be a simple graph with $\mathrm{p}$ vertices and $\mathrm{q}$ edges. Let $f: \mathrm{V}(\mathrm{G}) \rightarrow\{0,1,2, \ldots \mathrm{q}\}$ is a one

to one mapping. $f$ is called graceful mapping if the induced mapping $f^{+}: \mathrm{E}(\mathrm{G}) \rightarrow\{1,2, \ldots \mathrm{q}\}$ defined by $f^{+}(\mathrm{uv})=\mid f(\mathrm{u})-$ $f(\mathrm{v}) \mid$ is also a one to one, onto mapping.

\subsection{Super Vertex Graceful Labelling}

Let $\mathrm{G}=(\mathrm{V}, \mathrm{E})$ be a simple graph with $\mathrm{p}$ vertices and $\mathrm{q}$ edges. Let $f: \mathrm{V}(\mathrm{G}) \rightarrow \mathrm{P}$ is a one to one mapping. $f$ is called super vertex graceful mapping if the induced mapping $f^{*}: \mathrm{E}(\mathrm{G}) \rightarrow \mathrm{Q}$ defined by $f^{+}(\mathrm{uv})=f(\mathrm{u})+f(\mathrm{v})$ is also a bijection, where

$$
\begin{gathered}
P=\left\{\begin{array}{l} 
\pm 1, \pm 2, \ldots \pm \frac{p}{2} \text { if } p \text { is even } \\
0, \pm 1, \pm 2, \ldots \pm \frac{p-1}{2} \text { if } p \text { is odd }
\end{array}\right. \\
Q=\left\{\begin{array}{l} 
\pm 1, \pm 2, \ldots \pm \frac{q}{2} \text { if } q \text { is even } \\
0, \pm 1, \pm 2, \ldots \pm \frac{q-1}{2} \text { if } q \text { is odd }
\end{array}\right.
\end{gathered}
$$

Consider the cycle $\mathrm{C}_{5}$ with vertices $\left\{v_{1}, v_{2}, \ldots v_{5}\right\}$. Since both the order and size of $C_{5}$ are 5 . Let $P=\{-2,-1,0,1,2\}=$ $Q$. Let $f: \mathrm{V}\left(\mathrm{C}_{5}\right) \rightarrow P$ such that $f\left(v_{1}\right)=-2 ; f\left(v_{2}\right)=$ $0 ; f\left(v_{3}\right)=2 ; f\left(v_{4}\right)=-1 ; f\left(v_{5}\right)=1$. Let $\mathrm{e}_{\mathrm{i}}$ be the edge connecting the vertices $v_{i}$ and $v_{i+1}, i=1,2,3,4$ and $e_{5}$ be the edge connecting $\mathrm{v}_{5}$ and $\mathrm{v}_{0}$. Then the mapping $f^{+}\left(e_{1}\right)=$ $-2 ; f^{+}\left(e_{2}\right)=2 ; f^{+}\left(e_{1}\right)=-2 ; f^{+}\left(e_{3}\right)=1 ; f^{+}\left(e_{5}\right)=-1$ with " $f$ " make $\mathrm{C}_{5}$ as super vertex graceful graph.

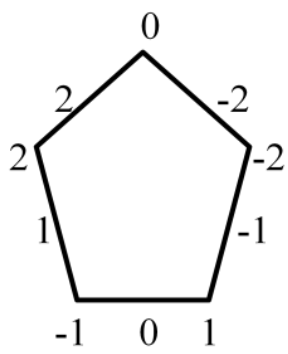

Fig. 1 A SVG of order 5 and size 5

As an another example, the following graph is also a super vertex graceful, where $\mathrm{P}=\{-3,-2,-1,1,2,3\}$ and $\mathrm{Q}=\{-4$, $3,-2,-1,1,2,3,4\}$

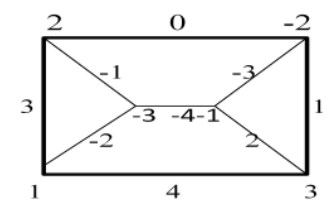

Fig. 2 A SVG of order 6 and size 8

\section{RESULTS}

\subsection{Lemma}

Let $\mathrm{G}$ be a graph with $\mathrm{p}$ vertices and $\mathrm{q}$ edges. If $\mathrm{p}$ is even, then $\max (\mathrm{Q})=\mathrm{p}-1 ; \min (\mathrm{Q})=-\mathrm{p}+1$.

Proof: If $\mathrm{p}$ is even, $P=\left\{ \pm 1, \pm 2, \ldots \pm \frac{p-2}{2}, \pm \frac{p}{2}\right\}$.
$\operatorname{Max}(\mathrm{Q})=\frac{p}{2}+\frac{p-2}{2}=p-1, \operatorname{Min}(\mathrm{Q})=-\frac{p}{2}-\frac{p-2}{2}=-p+1$.

\subsection{Theorem}

Let $G$ be a graph with $p$ vertices. If $G$ is super vertex graceful then the size of $G$ is utmost $2 p-1$ if $p$ is even and $2 p-3$ if $p$ is odd.

Proof:

Case(i)Let $\mathrm{p}$ is even. Then $\mathrm{G}$ has atmost $\frac{p(p-1)}{2}$ edges. In this case $P=\left\{ \pm 1, \pm 2, \ldots \pm \frac{p-2}{2}, \pm \frac{p}{2}\right\}$. Then by the lemma 3.1, the set $\mathrm{Q}$ has maximum value $\frac{p}{2}+\frac{p-2}{2}=p-1$ and minimum

Examples 
value $-(p-1)$. Hence $|\mathrm{G}|$ has atmost $2(\mathrm{p}+1)+1=2 \mathrm{p}-1$, elements including the edge with label 0 .

Case(ii) Let $\mathrm{p}$ be odd. Then $P=\left\{0, \pm 1, \pm 2, \ldots \pm \frac{p-1}{2}\right\}$. Then $\mathrm{Q}$ has maximum value $\mathrm{p}-2$ and minimum value $-(\mathrm{p}-2)$. Hence $|\mathrm{G}|$ has atmost $2(p-2)+1=2 p-3$ elements. Hence the theorem.

\section{Example}

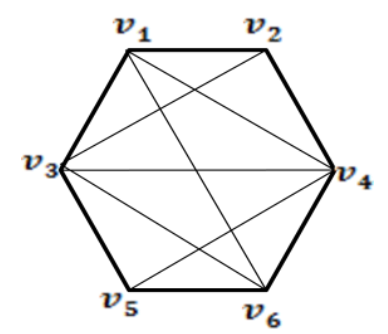

Fig. 3 A graph of order 6 and size 12

Here $\mathrm{q}=2 \mathrm{p}-1, \mathrm{p}=6$ (even). Consider the graph given in fig. 3 with vertices $\left\{v_{1}, v_{2}, v_{3}, v_{4}, v_{5}, v_{6}\right\}$. Then by the definition of super vertex graceful map $P=\{-3,-2,-1,1,2,3\}$. The size of $\mathrm{G}$ is 12 . Then $Q=\{-6,-5,-4,-3,-2,-1,0,1,2$, $3,4,5,6$. If $f v 3=3$ and $f v 4=2$, then $f+v 3, v 4=3+2=5$. Hence the induced map $f^{+}$can induce only the values $1,2,3$, $4,5,-1,-2,-3,-4,-5$. The remaining 2 edges cannot be labelled.

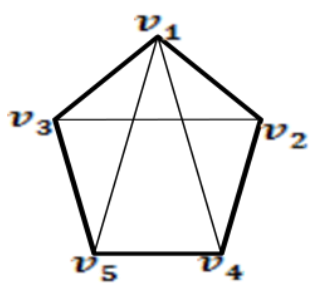

Fig. 4 A graph of order 5 and size 8

If $\mathrm{q}=2 \mathrm{p}-3, \mathrm{p}=5$ (odd). Then $P=\{-2,-1,0,1,2\}$. The size of the graph is $Q=\{-4,-3,-2,-1,0,1,2,3,4\}$. If $f\left(v_{1}\right)=1$ and $f\left(v_{2}\right)=2$, then $f^{+}\left(v_{1}, v_{2}\right)=1+2=3$. The labels $4,-4$ cannot be induced by the map $f^{+}$. $\therefore$ the graph is not super vertex graceful.

\subsection{Lemma}

$\frac{n(n-1)}{2}$ is even when $\mathrm{n}$ is a multiple of 4 .

\section{Proof:}

Let us prove this lemma by induction principle on n.If $\mathrm{n}=4$, $\frac{n(n-1)}{2}=\frac{4 x 3}{2}=6$ which is even. Let $\mathrm{n}=\mathrm{k}$, be a multiple of 4 and $\frac{k(k-1)}{2}$ is an even number.ie $\frac{k(k-1)}{2}=2 m \Rightarrow k^{2}-k=$ 4m. $\therefore k^{2}=4 m+k$. For $\mathrm{n}=\mathrm{k}+1, \quad \frac{(k+1) k}{2}=\frac{k^{2}+k}{2}=$ $\frac{4 m+k+k}{2}=\frac{4 m+2 k}{2}=2 m+k$ which is also an even number.Hence by induction principle, it can be concluded that $\frac{n(n-1)}{2}$ is even for all multiples of 4 .

\subsection{Lemma}

For $(i) n \geq 5,2 n-1<\frac{n(n-1)}{2}$; (ii) $2 n-3<\frac{n(n-1)}{2}$.

Proof:
We prove that (i), then (ii) is obvious. We use induction on $n$ to prove (i). If $\mathrm{n}=5,2 \mathrm{n}-1=9, \frac{n(n-1)}{2}=10$. So, let $2 k-1<$ $\frac{k(k-1)}{2}$. We prove $2(k+1)-1<\frac{(k+1) k}{2}$.

Now $2(\mathrm{k}+1)-1=2 \mathrm{k}+2-1=(2 \mathrm{k}-1)+2<\frac{k(k-1)}{2}+2=\frac{k^{2}-k+4}{2}<$ $\frac{k^{2}+k}{2}=\frac{k(k+1)}{2}$.

\subsection{Theorem}

Let $\mathrm{G}=(\mathrm{V}, \mathrm{E})$ be a super vertex graceful graph with even number of vertices and even number of edges. Then there is no vertex $\mathrm{u}$ in $\mathrm{V}$, such that $\operatorname{deg}(\mathrm{u})=|\mathrm{V}|-1$.

Proof:

If possible assume that there is $u$ in $\mathrm{V}$ such that $\operatorname{deg}(\mathrm{u})=|\mathrm{V}|$ 1. Also assume that the vertex $\mathrm{u}$ is labelled with integer $i \leq$ $\frac{|V|}{2}$. Let $\mathrm{f}(\mathrm{u})=i \leq \frac{|V|}{2}$. Since $\mathrm{G}$ is super vertex graceful graph, there must be a vertex $\mathrm{v}$ in $\mathrm{V}$ such that $\mathrm{v}$ is labelled with the integer $-\mathrm{i}$ i.ef( $(\mathrm{v})=-\mathrm{i}$. Moreover the vertex $\mathrm{u}$ is adjacent to every other vertex in G. In particular $\mathrm{u}$ is adjacent to v. Hence the induced map $f^{+}$gives us that $f^{+}(\mathrm{uv})=f(\mathrm{u})+f(\mathrm{v})=0$. Since $G$ has even number of edges, $0 \notin Q$.This is a contradiction. Hence there is no $u$ in V such that $\operatorname{deg}(\mathrm{u})=|\mathrm{V}|$ 1.

\subsection{Lemma}

The complete graphs $\mathrm{K}_{2}, \mathrm{~K}_{3}$ are super vertex graceful graphs. Proof:

Let $\mathrm{V}\left(\mathrm{K}_{2}\right)=\{\mathrm{u}, \mathrm{v}\}$, and $\mathrm{E}\left(\mathrm{K}_{2}\right)=\mathrm{e} . \mathrm{P}=\{-1,1\}$ and $\mathrm{Q}=\{0\} \cdot \mathrm{K}_{2}$ is the graph with one edge and two vertices $u$ and $v$. The edge $e$ is incident with the vertices $\mathrm{u}$ and v. $f$ is a map from $\mathrm{V} \rightarrow$ $P$ and if $f(u)=-1, f(v)=1$ and $f^{+}(\mathrm{e})=f^{+}(\mathrm{uv})=f(\mathrm{u})+f$ $(\mathrm{v})=1+(-1)=0$. Hence $\mathrm{K}_{2}$ is super vertex graceful.

Let $\mathrm{V}\left(\mathrm{K}_{3}\right)=\{\mathrm{x}, \mathrm{y}, \mathrm{z}\}, \mathrm{E}\left(\mathrm{K}_{3}\right)=\left\{\mathrm{e}_{1}, \mathrm{e}_{2}, \mathrm{e}_{3}\right\}$.Here $\mathrm{P}=\{-1,0,1\}$ and $\mathrm{Q}=\{-1,0,1\} . f$ is a map from $\mathrm{V} \rightarrow P$ and if $f(x)=-1, f(y)=$ $1, f(z)=0$. The edge $\mathrm{e}_{1}$ is incident with $\mathrm{x}$ and $\mathrm{y}, \mathrm{e}_{2}$ is incident with $\mathrm{y}$ and zand $\mathrm{e}_{3}$ is incident with $\mathrm{x}$ and $\mathrm{z}$. Then $f^{+}\left(\mathrm{e}_{1}\right)=f^{+}(\mathrm{xy})$ $=f(\mathrm{x})+f(\mathrm{y})=1+(-1)=0, f^{+}\left(\mathrm{e}_{2}\right)=f^{+}(\mathrm{yz})=f(\mathrm{y})+f(\mathrm{z})=1+$ $0=1: f^{+}\left(\mathrm{e}_{3}\right)=f^{+}(\mathrm{xz})=f(\mathrm{x})+f(\mathrm{z})=1+0=1$. Hence $\mathrm{K}_{3}$ is super vertex graceful.

\subsection{Theorem}

The complete graphs $\mathrm{K}_{4 \mathrm{n}} ; \mathrm{n}=1,2,3 \ldots$ is not a super vertex graceful graph.

\section{Proof:}

From the theorem 3.5, it can be concluded that the number of edges is $\mathrm{K}_{4 \mathrm{n}}$ is even for all values of $n$. Hence $\mathrm{K}_{4 \mathrm{n}}$ has even number of vertices and even number of edges. Also, for every $u \in\left|V\left(K_{4 n}\right)\right|-1$. Therefore from the above theorem, $\mathrm{K}_{4 \mathrm{n}}$ cannot be super vertex graceful.

Example

Consider the graph $\mathrm{G}=\mathrm{K}_{4 \mathrm{n},} \mathrm{n}=3$. Then the vertex set $V=\left\{v_{1}, v_{2}, \ldots, v_{12}\right\}$ and hence $P=\{ \pm 1, \pm 2, \pm 3, \pm 4, \pm 5$, $\pm 6\}$. The number of edges is 66 . Then $Q=\{ \pm 1, \pm 2, \ldots \pm 33\}$. In $\mathrm{K}_{\mathrm{n}} d\left(v_{i}\right)=n-1$ for all $i$. If $f\left(v_{k}\right)=j$ for $j \in P$. The vertex $\mathrm{v}_{\mathrm{k}}$ is adjacent to the remaining 11 vertices of $\mathrm{V}$ For some $\mathrm{r}, f\left(v_{r}\right)=-j$. Then the induced map $f^{+}\left(v_{k}, v_{r}\right)=$ $f\left(v_{k}\right)+f\left(v_{r}\right)=j-j=0 \notin Q . \therefore$ is not super vertex graceful.

\subsection{Theorem}

Complete bipartite graph $\mathrm{K}_{(1,2 \mathrm{n})}$ is a super vertex graceful for all values of $n$.

Proof: 
Let $\mathrm{G}=\left\{\mathrm{V}, \mathrm{E}, \mathrm{f}, \mathrm{f}^{+}\right\}$be a complete bipartite graph. The order and size of $K_{(1,2 n)}$ is $2 n+1$ and $2 n$. Let $V=\left\{V_{1}, V_{2}\right\}$ where $V_{1}$ and $\mathrm{V}_{2}$ are the partitions of $\mathrm{V}$. The complete bipartite graph is obtained by joining the vertices $V_{1}$ with vertices of $V_{2}$. $f$ is a bijective mapping from $\mathrm{V}$ to $\mathrm{P}$ and $f^{+}$is bijective map from $\mathrm{E}$ to $\mathrm{Q}$ as follows: $f^{+}(\mathrm{u}, \mathrm{v})=f(\mathrm{u})+f(\mathrm{v})$, where $\mathrm{P}$ and $\mathrm{Q}$ are the vertex label and edge label sets respectively and are given by $\mathrm{P}=\{0, \pm 1, \pm 2, \cdots, \pm n\}$ and $\mathrm{Q}=\{ \pm 1, \pm 2, \cdots, \pm n\}$. Let the set $\mathrm{P}$ be partitioned into two sets $\mathrm{P}_{1}$ and $\mathrm{P}_{2}$ such that $\mathrm{P}=\mathrm{P}_{1} \cup \mathrm{P}_{2}$ and $P_{1} \cap P_{2}=\Phi$. i.e. $P_{1}=0 ; P_{2}=\{ \pm 1, \pm 2, \ldots, \pm n\}$. Now we define the induced map $f^{+}$by

$\stackrel{+}{f}\left(\mathrm{u}, \quad \mathrm{v}_{\mathrm{i}}\right)=f(\mathrm{u})+f\left(\mathrm{v}_{\mathrm{i}}\right)=\{-\mathrm{n},-(\mathrm{n}-1), \ldots-1,1,2, \ldots,(\mathrm{n}-1)$, $\mathrm{n}$ \}. Therefore the map fis a SVG map for all values of ' $n$ ' and hence $\mathrm{K}_{(1,2 \mathrm{n})}$ is a SVG.

\subsection{Theorem}

The complete bipartite graph $\mathrm{K}_{2,2 \mathrm{n}+1}$ is super vertex graceful. Proof:

Let $G=\left\{V, E, f, f^{+}\right\}$be a bipartite graph. The order of $K_{(2,2 n-1)}$ is $2 n+1$ and size is $4 n-2$. The vertex label set $P=\{0, \pm 1, \pm 2$, $\cdots, \pm \mathrm{n}\}$ and the edge label set $\mathrm{Q}=\{0, \pm 1, \pm 2, \cdots, \pm 2 \mathrm{n}-1\}$. The vertex set is partitioned into two sets $V_{1}$ and $V_{2}$ where $V_{1}$ $=\left\{\mathrm{x}_{1}, \mathrm{x}_{2}\right\} \mathrm{V}_{2}=\left\{\mathrm{x}_{3}, \mathrm{x}_{4}, \cdots, \mathrm{x}_{2 \mathrm{n}+1}\right\}$. The map fi : Vi $\rightarrow \mathrm{Pi}$ is a bijective map. The map $\mathrm{f}^{+}: \mathrm{E} \rightarrow \mathrm{Q}$ is also a bijective map defined as $\stackrel{+}{f}(\mathrm{u}, \mathrm{v})=f(\mathrm{u})+f(\mathrm{v})$. Here $\mathrm{P}_{1}=\{ \pm \mathrm{n}\}$ and $\mathrm{P}_{2}=\{0, \pm 1$, $\pm 2 \cdots \pm(\mathrm{n}-1)\}$. Then $\mathrm{G}$ is a SVG graph with $f^{+}$as SVG mapping.

\section{DISCUSSION}

The labelling of graphs under super vertex graceful map depends on the order and size. Similar analysis can be done for other mapping and some particular graphs.

\section{REFERENCES}

[1] B.D. Acharya, Construction of certain infinite families of graceful graphs from a given graceful graph, Def Sci J, Vol 32, No 3,July 1982, PP 231-236.

[2] Bondy J. A. and. Murty, U.S.R Graph Theory with applications, Newyork Macmillan Ltd. Press,1976.

[3] Brundage, M. "Graceful Graphs" http:// www.qbrundage.com/ ichael/pubs/graceful/.
[4] Frank Van Russel, "Relaxed Graceful Labelling of Trees", The electronic Journal of Combinatorics , 2002.

[5] Golomb, http:// Graceful graph/ Labeled Graphs/ Graph theory/ Discrete Mathematics/Math forum.

[6] Harary, Graph Theory, Narosa Publishing House, 2001.

[7] Joseph A. Gallian, A Dynamic survey of Graph Labeling, 2008.

[8] Juraj Bosak, Decomposition of graphs, Kluwer Academic Publishers 1990.

[9] Murugesan. N, Uma. R, A Conjecture on Amalgamation of graceful graphs with star graphs, Int.J.Contemp.Math.Sciences, Vol.7, 2012, No.39, 19091919.

[10] Murugesan. N, Uma.R, Super vertex gracefulness of complete bipartite graphs, International J.of Math.Sci \& Engg. Appls, Vol.5, No.VI (Nov, 2011), PP 215-221.

[11] Murugesan. N, Uma. R, Graceful labeling of some graphs and their subgraphs, Asian Journal of Current Engineering and Maths1:6 Nov - Dec (2012) 367 - 370.

[12] Murugesan. N, Uma.R Fibonacci gracefulness of $\mathrm{P}_{\mathrm{n}}{ }^{2}$ and PP @SQ , International J. of Math. Sci. \& Engg. Appls, , Vol. 7 No. IV (July, 2013), pp. 429-437.

[13]Murugesan.N,Uma.R, Super vertex gracefulness of some cycle-related graphs, Proceedings of the international conference on mathematical methods and computation, 2014.

[14] A Rosa, On certain valuations of the vertices of a graph, theory Of Graphs (Internet. Sympos., Rome, 1996), Gordon and Breach, Newyork, 1967, pp. 349-355.

[15] Sin - Min - Lee, Elo Leung and Ho Kuen Ng, On Super vertex graceful unicyclic graphs, Czechoslovak mathematical Journal, 59 (134) (2009), 1- 22.

[16] Solairaju. A, Vimala. C, Sasikala. A, Edge - Odd gracefulness of $\mathrm{P}_{\mathrm{M}} \Theta \mathrm{S}_{\mathrm{N}}$, for $\mathrm{M}=5,6,7,8$, International Journal of Computer applications (0975 - 8887), Volume 9- No. 12, November 2010. 